\title{
Blind Channel Equalization Using Constrained Generalized Pattern Search Optimization and Reinitialization Strategy
}

\author{
Abdelouahib Zaouche, ${ }^{1}$ lyad Dayoub, ${ }^{2}$ Jean Michel Rouvaen, ${ }^{2}$ and Charles Tatkeu ${ }^{1}$ \\ ${ }^{1}$ INRETS LEOST, 20 rue Elisee Reclus, 59650 Villeneuve d'Ascq, France \\ ${ }^{2}$ IEMN DOAE, University of Valenciennes and Hainaut-Cambresis, Le Mont Houy, 59313 Valenciennes, France
}

Correspondence should be addressed to Abdelouahib Zaouche, abdelouahib.zaouche@inrets.fr

Received 5 November 2007; Revised 28 May 2008; Accepted 26 August 2008

Recommended by William Sandham

We propose a global convergence baud-spaced blind equalization method in this paper. This method is based on the application of both generalized pattern optimization and channel surfing reinitialization. The potentially used unimodal cost function relies on higher- order statistics, and its optimization is achieved using a pattern search algorithm. Since the convergence to the global minimum is not unconditionally warranted, we make use of channel surfing reinitialization (CSR) strategy to find the right global minimum. The proposed algorithm is analyzed, and simulation results using a severe frequency selective propagation channel are given. Detailed comparisons with constant modulus algorithm (CMA) are highlighted. The proposed algorithm performances are evaluated in terms of intersymbol interference, normalized received signal constellations, and root mean square error vector magnitude. In case of nonconstant modulus input signals, our algorithm outperforms significantly CMA algorithm with full channel surfing reinitialization strategy. However, comparable performances are obtained for constant modulus signals.

Copyright (C) 2008 Abdelouahib Zaouche et al. This is an open access article distributed under the Creative Commons Attribution License, which permits unrestricted use, distribution, and reproduction in any medium, provided the original work is properly cited.

\section{INTRODUCTION}

The major problem encountered in digital communications is intersymbol interference (ISI). The received signal is seriously distorted, due to the band limiting effect of the channel and the multipath propagation phenomenon. To overcome such problems, various channel equalization techniques have been proposed over the past few years. Most of these techniques take advantage of known training sequences to adaptively extract channel information. The main drawback with this approach is bandwidth consuming due to training. To overcome this resource wasting, blind equalization algorithms have been proposed. In this case, instead of using training sequences, only input signal and noise statistical properties are required. Thus, the original transmitted message is recovered from the received sequence that is corrupted by noise and ISI with no training sequence nor a priori channel knowledge [1]. In general, the blind equalization techniques can be classified according to their signals statistical properties exploitation, as those using maximum likelihood (ML) methods [2], or second-order statistics (SOS) [3] or higher-order statistics (HOS) [1,
4]. The latter include inverse filter criteria-(IFC-) based algorithms [5], the super exponential algorithm (SEA) [6], polyspectra-based algorithm [7], and Bussgang algorithms [8]. Blind equalization based on higher-order statistics relies mainly on the optimization of nonlinear and nonconvex cost functions. These cost functions have a highly multimodal geometrical structure with many local minima $[9,10]$. This fact makes the global optimization task very tedious. Nowadays many digital communication schemes transmit constant modulus (CM) signals. Hence, several iterativegradient-based blind equalization algorithms exploiting this precious information, namely, the constant modulus criterion, have been developed and have gained a widespread use in different communication systems [9]. Among these algorithms, the constant modulus algorithm (CMA) is the most commonly used. Moreover, it is reputed to be the simplest and most successful HOS-based blind equalization algorithm $[4,9,10]$. However, the multimodal structure of the nonconvex and nonlinear CM fitness function makes these algorithms extremely vulnerable to converge toward local minima. This leads to formulate the problem of blind equalization as a constrained gradient-free optimization 


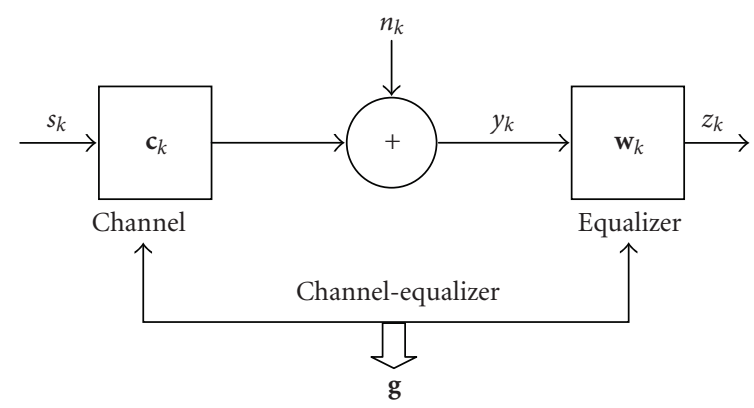

FIgURE 1: Block diagram of the system.

problem using generalized pattern search algorithm that minimizes $\|\mathbf{g}\|_{2}^{4}-\|\mathbf{g}\|_{4}^{4}$ over a search space, where $\mathbf{g}$ is the joint channel-equalizer impulse response. This cost function is known to be potentially unimodal as discussed in $[11,12]$; however, this is not sufficient to warrant global convergence. To overcome this limitation and to ensure good global convergence behavior, we propose to use the channel surfing reinitialization strategy to estimate the optimal delay.

\section{SYSTEM MODEL}

The block diagram of the system under consideration is shown in Figure 1. It represents a single-input-single-output (SISO) channel equalizer. The source sequence $\left\{s_{k}\right\}$, with finite real or complex alphabet, is assumed to be subGaussian (kurtosis $<3$ if real or kurtosis $<2$ if complex), circular $\left(E\left\{s_{k}^{2}\right\}=0\right.$ in the complex case), independent, and identically distributed (i.i.d) with variance $E\left\{\left|s_{k}\right|^{2}\right\}=\sigma_{s}^{2}$. This sequence is transmitted through a complex linear time invariant baseband channel represented by a discrete finite impulse response (FIR) filter $\mathbf{c}$ of length $P$ as

$$
\mathbf{c}=\left[\begin{array}{llll}
c_{0} & c_{1} & \cdots & c_{P-1}
\end{array}\right]^{T},
$$

the $c_{k}$ 's being complex numbers.

The resulting signal is corrupted by a zero-mean random Gaussian noise $\left\{n_{k}\right\}$ with variance $\sigma_{n}^{2}$, independent of the input source sequence $\left\{s_{k}\right\}$, resulting in the regressor sequence $\left\{y_{k}\right\}$. The latter is then processed by an $L$-taped FIR blind equalizer with complex coefficients $\mathbf{w}$, given by

$$
\mathbf{w}=\left[\begin{array}{llll}
w_{0} & w_{1} & \cdots & w_{L-1}
\end{array}\right]^{T} .
$$

The goal of the blind equalizer is to provide an accurate estimate of the transmitted sequence, that we denote by $\left\{z_{k}\right\}$. This is achieved when the combined channel-equalizer impulse response $\mathbf{g}=\mathbf{c} \otimes \mathbf{w}(\otimes$ meaning convolution) behaves as a simple delay operator resulting in $\left\{z_{k}\right\} \approx\left\{s_{k-\delta}\right\}$. This is referred to as the zero forcing condition which can be measured using the intersymbol interference (ISI) formula defined in terms of the global channel-equalizer taps as

$$
\text { ISI }=\frac{\left(\sum_{i}\left|g_{i}\right|^{2}\right)-|\mathbf{g}|_{\max }^{2}}{|\mathbf{g}|_{\max }^{2}},
$$

where $|\mathbf{g}|_{\max }$ stands for the maximum joint channelequalizer filter weight in absolute value.

Furthermore, it can be noticed that the zero forcing condition corresponds ideally to an ISI equal to zero. Thus, blind equalization can be viewed as the minimization of the above ISI.

\section{PROBLEM FORMULATION}

Most of blind equalization algorithms rely on the minimization of nonconvex cost functions. Among these algorithms, constant modulus algorithm (CMA) is the most commonly used blind equalization scheme. It is mainly based on the use of stochastic gradient descent (SGD) strategy to minimize the nonconvex Godard's cost function defined by $J=E\{(\gamma-$ $\left.\left.\left|z_{k}\right|^{2}\right)^{2}\right\}$, where $\gamma=E\left\{\left|s_{k}\right|^{4}\right\} / E\left\{\left|s_{k}\right|^{2}\right\}$ is the dispersion constant $[13,14]$. However, the use of such nonconvex cost functions may result in undesirable convergence problems due to the presence of several local minima and saddle points. To overcome this limitation, many zero forcing blind equalization cost functions have been proposed in the literature [12-15]. In this paper, we use one of them, which is simple in implementation and gives the best performances. This cost function is expressed in terms of joint channel equalizer impulse response as

$$
\|\mathbf{g}\|_{2}^{4}-\|\mathbf{g}\|_{4}^{4}=\left(\sum_{i}\left|g_{i}\right|^{2}\right)^{2}-\sum_{i}\left|g_{i}\right|^{4} .
$$

It has been shown in $[13,14]$ that by employing the gradient of the cost function with respect to $g_{i}$ and setting it to zero, the corresponding extrema are the solutions of the following equation:

$$
\left(\sum_{i}\left|g_{i}\right|^{2}-\left|g_{i}\right|^{2}\right) g_{i}=0
$$

Note that (5) has two different solutions, one of which corresponds to $\mathbf{g}=0$. This undesirable solution may be avoided by imposing a linear constraint on the equalizer weights. Among the linear constraints proposed in the literature, we can cite the normalization of the blind equalizer weights $\mathbf{w}^{\prime}=\mathbf{w} / \sqrt{\mathbf{w}^{H} \mathbf{w}}$ after each iteration or, as suggested in [16], the assessment of a linear constraint of the form

$$
\mathbf{u}^{T} \mathbf{w}=e \quad \text { with } \mathbf{u} \neq \mathbf{0}, e \neq 0 .
$$

However, in order to reduce the computation complexity and to avoid the division by zero, here we use a linear constraint. This latter consists in setting one tap of the blind equalizer (say at index $\delta$ ) to zero. This is formulated as

$$
\text { minimize } f(\mathbf{w})=\|\mathbf{g}\|_{2}^{4}-\|\mathbf{g}\|_{4}^{4} \text { subject to } w_{\delta}=1,
$$

where $0 \leq \delta \leq L-1$.

The second solution of (5) corresponds to the desired zero forcing condition in which at most one nonzero element 


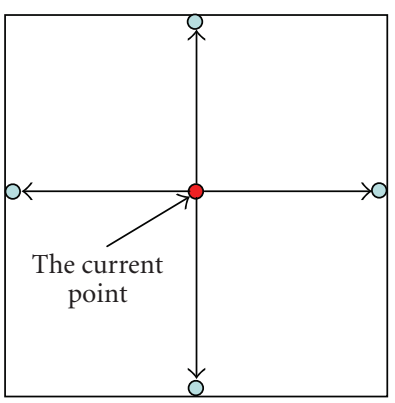

(a)

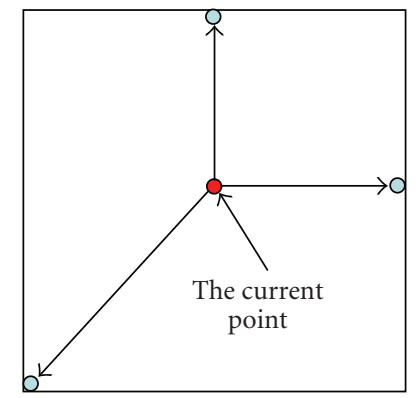

(b)
FIGURE 2: Pattern vectors for the (a) GPS $2 N=4$ positive basis and (b) GPS $N+1=3$ positive basis.

of the joint channel-equalizer impulse response $\mathbf{g}$ is allowed and the remaining taps are ideally forced to zeros. The use of a linear constraint on the equalizer tap directly rather than on $\mathbf{g}$ is due to two factors. The first one concerns the unavailability of $\mathbf{g}$. The second factor is motivated by the direct relationship between maximizing one tap of $\mathbf{g}$ located at any position $\delta$ and maximizing the equalizer tap located at the same position.

As discussed in [12], in the case of baud-spaced equalization (BSE), the cost function $\|\mathbf{g}\|_{2}^{4}-\|\mathbf{g}\|_{4}^{4}$ is sectionally convex in $\mathbf{g}$ and unimodal both in $\mathbf{g}$ and $\mathbf{w}$ for infinite length equalizers $(L=\infty)$. Moreover, in most practical situations corresponding to BSE with finite $N$, the optimization problem of (7) is a unimodal one in $\mathbf{g}$ for a given delay $\delta$ and potentially unimodal in $\mathbf{w}$. Thus, unimodality in $\mathbf{w}$, which ensures a global convergence, is not necessarily obtained. Unimodality in $\mathbf{w}$ for finite length BSE remains still unproven.

Godard's cost function has many local minima and saddle points for a given delay. Since the proposed cost function has a unique minimum in $\mathbf{g}$ for a given delay, it is less likely to have many local minima in w, unlike Godard's. However, the problem of global convergence still rises. Thus, in order to overcome this limitation, we propose to use the channel surfing reinitialization (CSR) strategy suggested in [17]. This has been originally proposed for CMA and we suggest to adapt here to the blind optimization problem of (7). CSR consists of varying the delay index $\delta$ systematically and searching the optimum equalizer $\mathbf{w}$ for each delay value. Finally, the optimum index which minimizes the cost function $f(\mathbf{w})$ is retained. In fact, unlike the CSR-CMA, where the algorithm parameters (step size and maximum number of iterations) must be adjusted for each value of $\delta$ to insure convergence, we propose to use CSR only to predict the global optimal delay index $\delta^{\dagger}$ for the blind equalizer tap which is fixed to one. First, for the sake of simplicity, we introduce a notation for the shift operator applied on any given vector $\mathbf{u}$ as

$$
\operatorname{SHIFT}_{K, n}(\mathbf{u}) \triangleq \mathbf{u}(n-K),
$$

where $K$ is an integer delay.
Let us also define the covariance matrix estimate of the preequalized data sequence $\left\{y_{k}\right\}$ as

$$
\widehat{\mathbf{R}} \triangleq E\left\{\mathbf{y}_{k} \mathbf{y}_{k}^{H}\right\} .
$$

As discussed in [17-19], if a Wiener equalizer for a particular delay has a reasonably good mean square error (MSE) performance in estimating $\left\{s_{k-\delta}\right\}$, there exists a blind equalizer in its immediate neighborhood. Using the converged blind equalizer $\mathbf{w}_{\delta}$ as an estimate of the MMSE equalizer results in the following estimate of the unknown channel impulse response $[17,18]$ :

$$
\widehat{\mathbf{c}}=\widehat{\mathbf{R}} \mathbf{w}_{\delta}
$$

A performance measure of the blind equalizer after convergence is the following estimate of the Wiener cost function:

$$
\hat{J}=1-\mathbf{w}_{\delta}^{H} \hat{\mathbf{c}}=1-\mathbf{w}_{\delta}^{H} \hat{\mathbf{R}} \mathbf{w}_{\delta} .
$$

The optimal delay is found using

$$
\delta^{\dagger}=\arg \min \{\underbrace{1-\left(\hat{\mathbf{R}}^{-1} \operatorname{SHIFT}_{\delta, k}(\hat{\mathbf{c}})\right)^{H} \operatorname{SHIFT}_{\delta, k}(\hat{\mathbf{c}})}_{\widehat{J}_{\delta, k}}\} .
$$

Therefore, the local optimization problem of (7) is transformed into the global blind equalization problem stated as

$$
\text { minimize } f(\mathbf{w})=\|\mathbf{g}\|_{2}^{4}-\|\mathbf{g}\|_{4}^{4} \text { subject to } w_{\delta^{\dagger}}=1 \text {. }
$$

It can be noticed that the optimization problem shown above is formulated in terms of the unknown joint channelequalizer impulse response g. Consequently, its implementation requires the formulation of the cost function only in terms of known quantities. These latter are the blind equalizer output sequence $\left\{z_{k}\right\}$ and the corresponding statistical measures related to the input source sequence $\left\{s_{k}\right\}$. It has been previously shown in [11] that

$$
f(\mathbf{w})=\left[\frac{E\left\{\left|z_{k}\right|^{2}\right\}}{E\left\{\left|s_{k}\right|^{2}\right\}}\right]^{2}-\frac{E\left\{\left|z_{k}\right|^{4}\right\}-3\left(E\left\{\left|z_{k}\right|^{2}\right\}\right)^{2}}{E\left\{\left|s_{k}\right|^{4}\right\}-3\left(E\left\{\left|s_{k}\right|^{2}\right\}\right)^{2}} .
$$

Using the definitions for the variance $\sigma_{s}^{2}$ and the normalized kurtosis $\kappa_{s}$ of the source sequence $\left\{s_{k}\right\}$ :

$$
\sigma_{s}^{2} \triangleq E\left\{\left|s_{k}\right|^{2}\right\}, \quad \kappa_{s} \triangleq \frac{E\left\{\left|s_{k}\right|^{4}\right\}}{\sigma_{s}^{4}},
$$

and those for the equalizer output statistics:

$$
E\left\{\left|z_{k}\right|^{2}\right\}=\frac{1}{N} \sum_{k=1}^{N}\left|z_{k}\right|^{2}, \quad E\left\{\left|z_{k}\right|^{4}\right\}=\frac{1}{N} \sum_{k=1}^{N}\left|z_{k}\right|^{4},
$$

where $N$ is the length of the sequence $\left\{z_{k}\right\},(14)$ may be written as

$$
f(\mathbf{w})=\frac{\xi \kappa_{s}}{N^{2}}\left(\sum_{k=1}^{N}\left|z_{k}\right|^{2}\right)^{2}-\frac{\xi}{N}\left(\sum_{k=1}^{N}\left|z_{k}\right|^{4}\right),
$$

where $\xi=1 /\left(\kappa_{s}-3\right) \sigma_{s}^{4}$. 


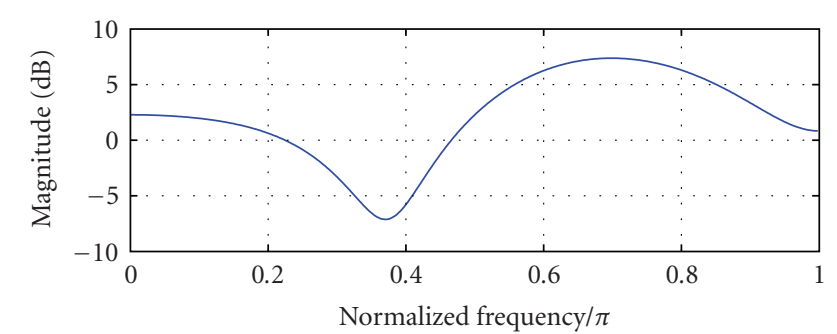

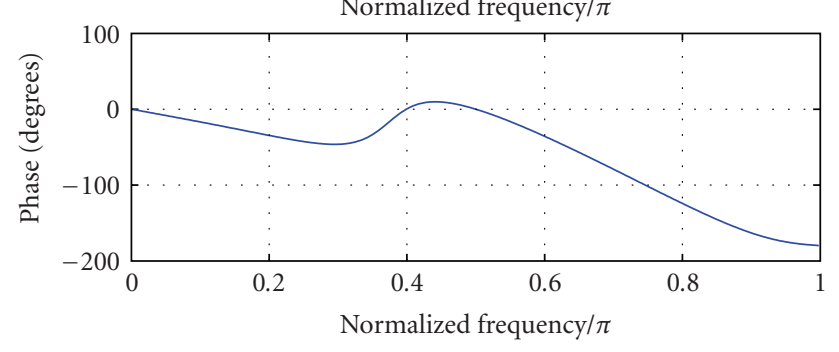

(a)

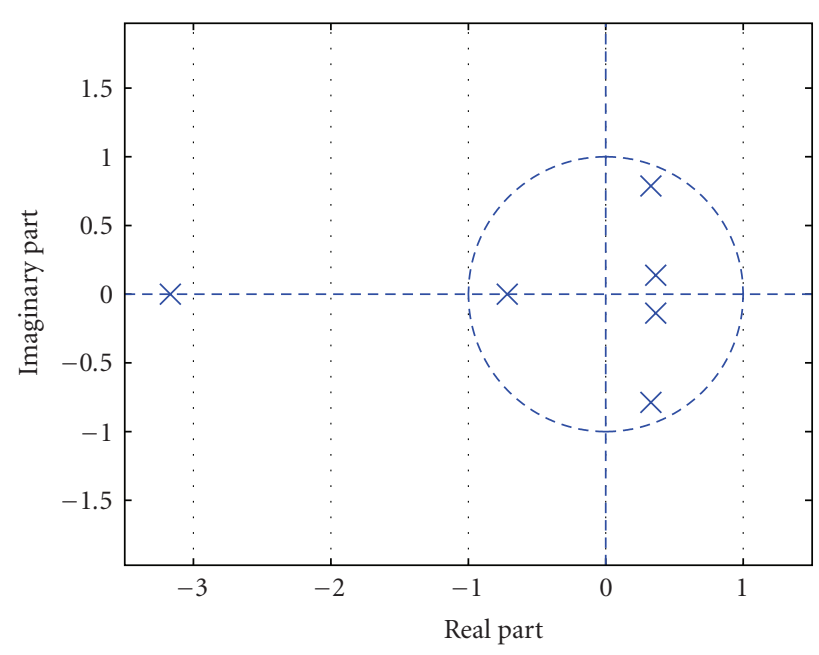

(b)

Figure 3: Example channel characteristics: (a) (top) frequency response, (a) (bottom) phase response, (b) and zeroes locations.

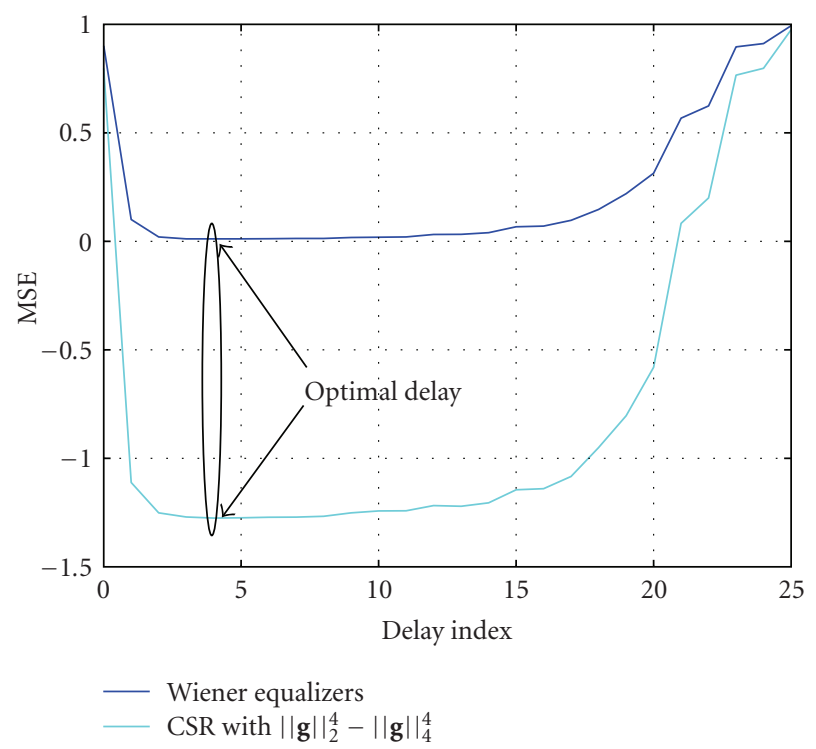

(a)

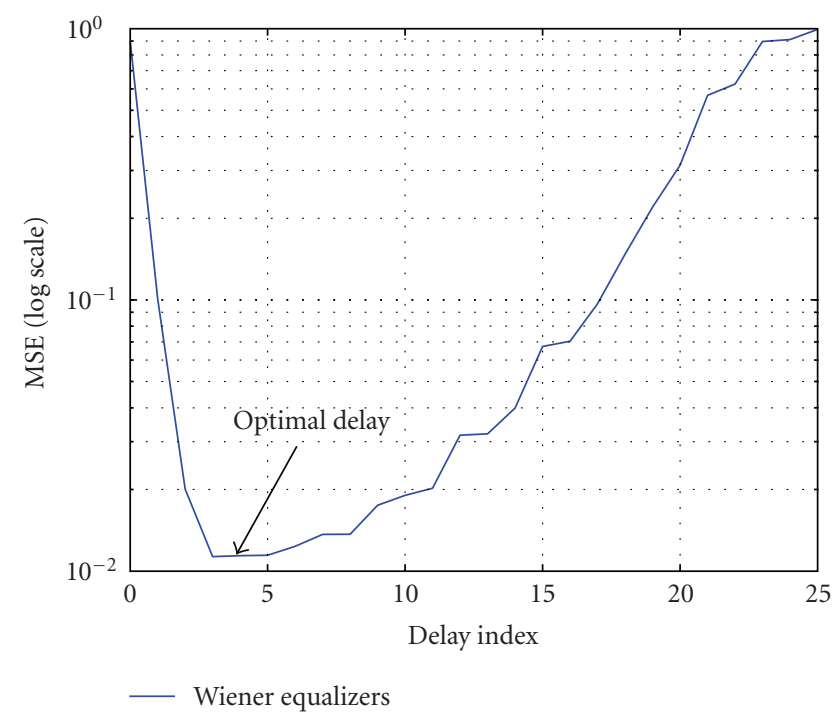

(b)

FIGURE 4: MSE versus system delays for (a) Wiener equalizers and logarithmic view for (b) Wiener equalizers.

Considering the digital communication system of Figure 1, the equalizer output $z_{k}$ can be expressed in terms of the unknown blind equalizer vector and the known regressor vector as

$$
z_{k}=\mathbf{w}^{H} \mathbf{y}_{k} .
$$

Substituting (18) into (17) yields the desired formulation of the cost function in terms only of the unknown blind equalizer vector and known statistical quantities as depicted below:

$$
f(\mathbf{w})=\frac{\xi \kappa_{s}}{N^{2}}\left(\sum_{k=1}^{N}\left|\mathbf{w}^{H} \mathbf{y}_{k}\right|^{2}\right)^{2}-\frac{\xi}{N}\left(\sum_{k=1}^{N}\left|\mathbf{w}^{H} \mathbf{y}_{k}\right|^{4}\right) .
$$

The proposed cost function deals with both real and complex channels and equalizers. In fact, the unknown blind equalizer is given by

$$
\mathbf{w}=\mathbf{w}_{\text {real }}+j \mathbf{w}_{\text {img }} .
$$

The effect of using complex equalizers rather than real ones resides in doubling the number of the unknown variables to be found by the optimization process.

Finally, in order to solve the optimization problem, the expression for $f(\mathbf{w})$ of (19) must now be substituted into (13).

The following section is dedicated to solving the above constrained optimization problem using generalized pattern search algorithm. 


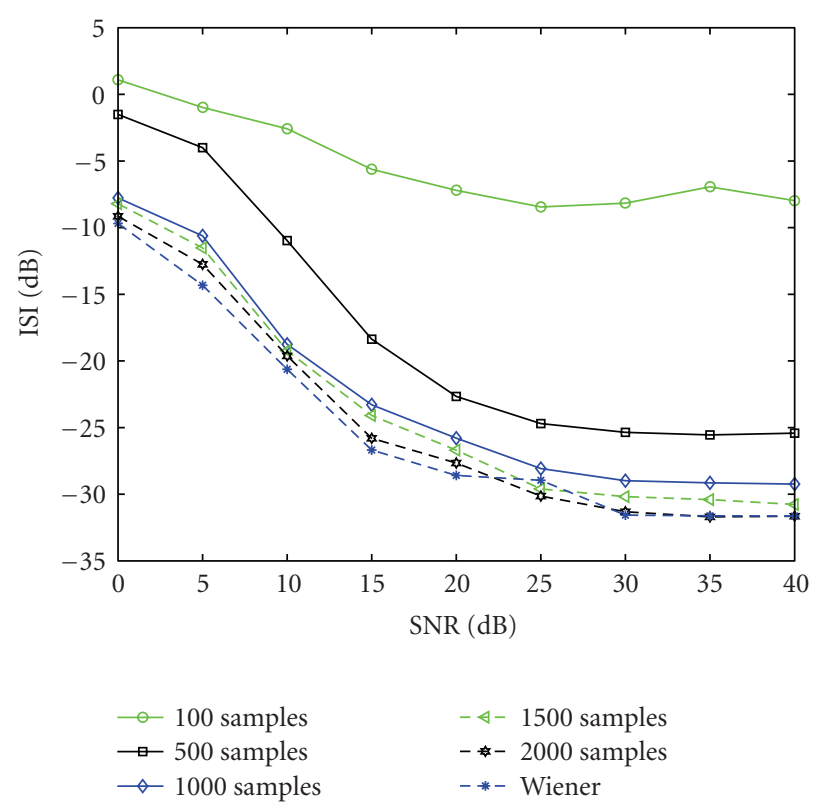

FIGURE 5: The proposed algorithm ISI performance for different sample sequence lengths and SNRs.

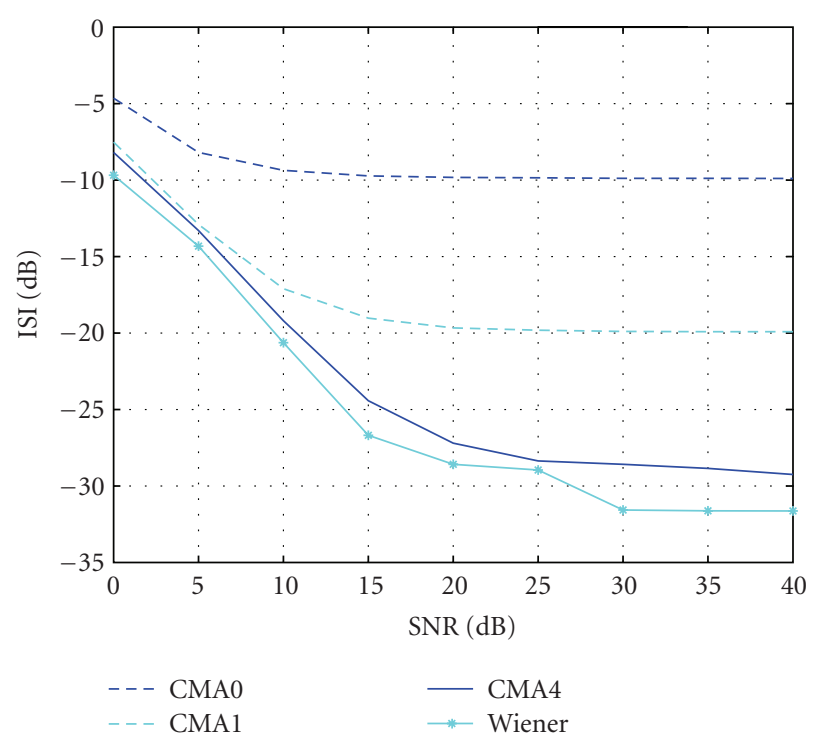

FIGURE 6: CMA ISI performance for different single spike initializations and SNRs.

\section{BACKGROUND ON GENERALIZED PATTERN SEARCH ALGORITHM}

Generalized pattern search (GPS) algorithms that were first defined and analyzed by Torczon [20] for derivative-free unconstrained optimization belong to the family of direct search methods. In fact they rely on searching for a set of points around the current point, forming a mesh, in order to find one fitness value lower than that at the current point. The essence of defining a mesh is to find a set of positive spanning directions $\mathbf{D}$ in $\mathfrak{R}^{n}$. To better understand the notion of positive spanning, we introduce the following definitions and terminology thanks to Davids [21].

Definition 1. A positive combination of the set of vectors $\mathbf{D}=$ $\left\{\mathbf{d}_{i}\right\}_{i=1}^{r}$ is a linear combination $\sum_{i=1}^{r} \alpha_{i} \mathbf{d}_{i}$, where $\alpha_{i} \geq 0, i=$ $1,2, \ldots, r$.

Definition 2. A finite set of vectors $\mathbf{D}=\left\{\mathbf{d}_{i}\right\}_{i=1}^{r}$ forms a positive spanning set for $\mathfrak{R}^{n}$, if every $\mathbf{v} \in \mathfrak{R}^{n}$ can be expressed as a positive combination of vectors in $\mathbf{D}$. The set of vectors $\mathbf{D}$ is said to positively span $\Re^{n}$. The set $\mathbf{D}$ is said to be a positive basis for $\mathfrak{R}^{n}$ if no proper subset of $\mathbf{D}$ positively spans $\mathfrak{R}^{n}$.

Davids demonstrated a very important feature, which proves determinant in the choice of the set of positive direction in GPS algorithms, namely, the cardinal of any positive set $\mathbf{D}$ in $\Re^{n}$, that we denote as $m$, lies between $n+1$ and $2 n$. This is mathematically formulated as

$$
n+1 \leq m=\operatorname{card}(\mathbf{D}) \leq 2 n,
$$

where the lower limit $n+1$ and upper limit $2 n$ stand for the cardinals of the minimal and maximal positive bases, respectively.

It is common to choose the positive bases as the columns of $\mathbf{D}_{\max }=\left[\mathbf{I}_{n \times n},-\mathbf{I}_{n \times n}\right]$ or $\mathbf{D}_{\min }=\left[\mathbf{I}_{n \times n},-\mathbf{e}_{n \times 1}\right]$, where $\mathbf{I}_{n \times n}$ is the $n \times n$ identity matrix and $\mathbf{e}_{n \times 1}$ is the $n$-dimensional column vector of ones $[22,23]$. As an example to highlight this point, let us consider that the blind equalization problem formulated using (13) is a two-dimensional one. This means that the unknown equalizer vector $\mathbf{w}$ has two taps $(n=2)$. According to (21), the cardinal of the positive basis to be used while applying GPS algorithm to the optimization problem lies between 3 and 4 . Indeed, the corresponding minimal positive basis having a cardinal of 3 is constructed of the column vectors of the matrix:

$$
\mathbf{D}_{\min }=\left[\mathbf{I}_{2 \times 2},-\mathbf{e}_{2 \times 1}\right]=\left[\begin{array}{ccc}
1 & 0 & -1 \\
0 & 1 & -1
\end{array}\right],
$$

yielding the following pattern search vectors:

$$
\mathbf{d}_{1}=\left[\begin{array}{ll}
1 & 0
\end{array}\right]^{T}, \quad \mathbf{d}_{2}=\left[\begin{array}{ll}
0 & 1
\end{array}\right]^{T}, \quad \mathbf{d}_{3}=\left[\begin{array}{ll}
-1 & -1
\end{array}\right]^{T} .
$$

Moreover, the corresponding maximal positive basis $\mathbf{D}_{\max }$ is then constructed as

$$
\mathbf{D}_{\max }=\left[\mathbf{I}_{2 \times 2},-\mathbf{I}_{2 \times 2}\right]=\left[\begin{array}{cccc}
1 & 0 & -1 & 0 \\
0 & 1 & 0 & -1
\end{array}\right],
$$

yielding

$$
\begin{aligned}
& \mathbf{d}_{1}=\left[\begin{array}{ll}
1 & 0
\end{array}\right]^{T}, \\
& \mathbf{d}_{2}=\left[\begin{array}{ll}
0 & 1
\end{array}\right]^{T}, \\
& \mathbf{d}_{3}=\left[\begin{array}{ll}
-1 & 0
\end{array}\right]^{T}, \\
& \mathbf{d}_{4}=\left[\begin{array}{ll}
0 & -1
\end{array}\right]^{T} .
\end{aligned}
$$




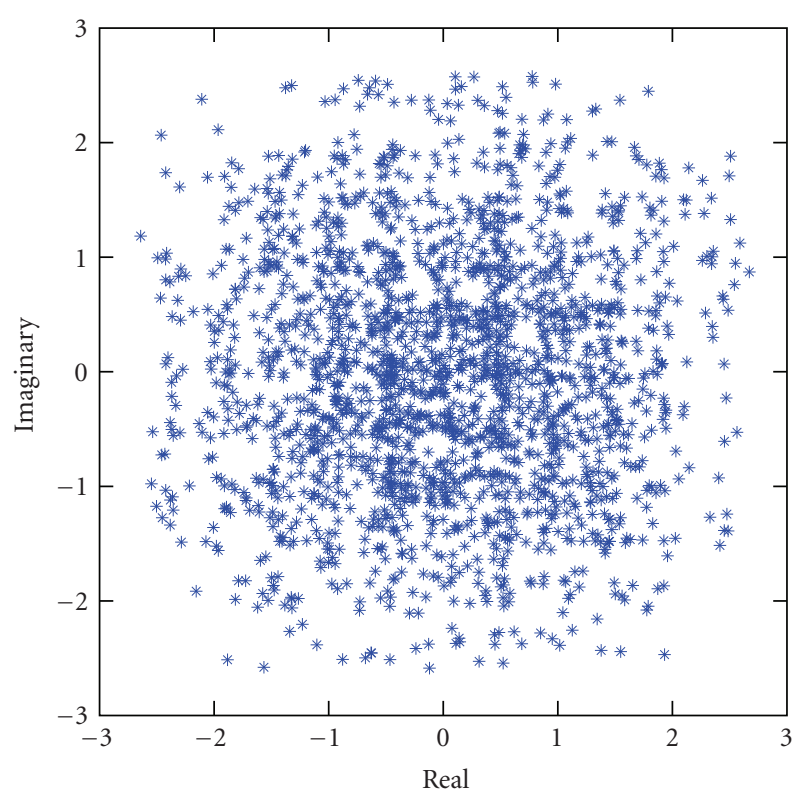

(a)

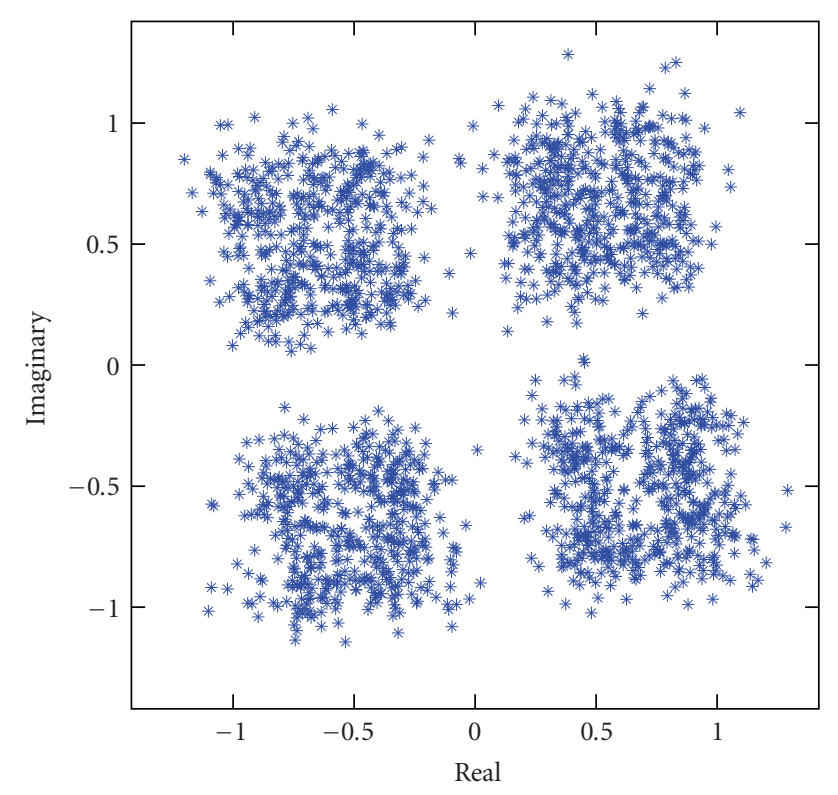

(c)

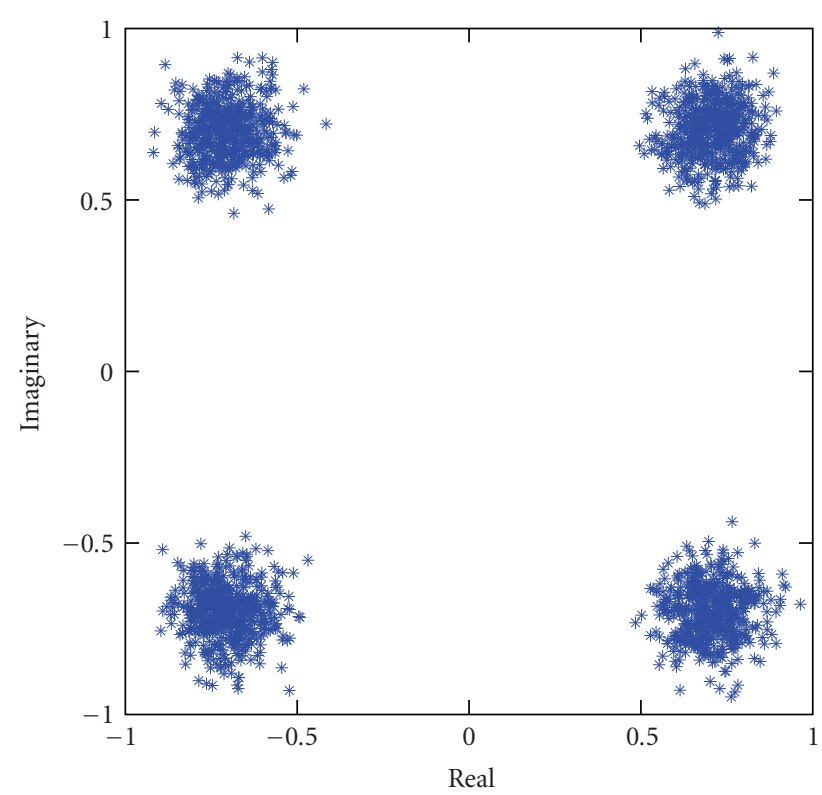

(b)

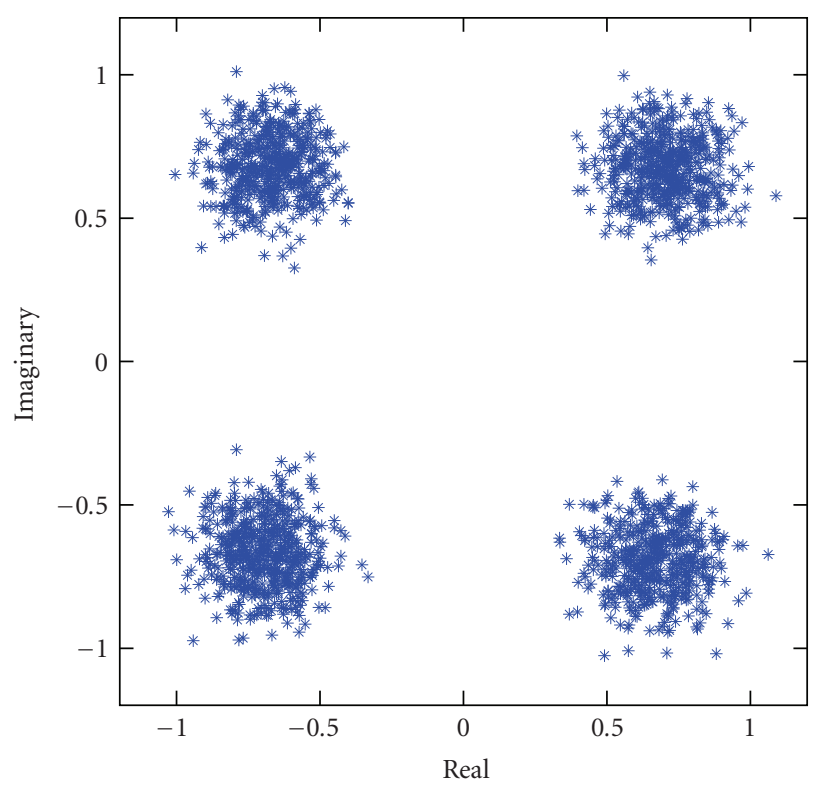

(d)

FIGURE 7: (a) QPSK constellations before equalization, (b) after equalization using GPS, (c) after equalization using CMA0, and (d) after equalization using CMA1.

These two minimal and maximal positive bases corresponding to the 2-dimensional optimization problem are illustrated in Figure 2. It is very important to point out the fact that the previous method of choosing the set of positive spanning directions is not unique.

In fact there is a great freedom in choosing these directions, but the set of positive directions $\mathbf{D}$ can be always expressed under the form $[24,25]$

$$
[\mathbf{D}]_{n \times m}=[\mathbf{G}]_{n \times n}[\mathbf{Z}]_{n \times m}
$$

where $\mathbf{G}$ is a nonsingular real generating matrix (most often taken as the identity matrix) and $\mathbf{Z}$ is a full rank integer matrix. Therefore, each direction vector $\mathbf{d}_{j} \in \mathbf{D}$ can be expressed as $\mathbf{d}_{j}=\mathbf{G} \mathbf{z}_{j}$, where $\mathbf{z}_{j}$ is an integer vector of length $n$.

\section{BLIND EQUALIZATION USING GPS ALGORITHM}

Generalized pattern search algorithms consist mainly of two phases: an optional search step and a local poll step. In 


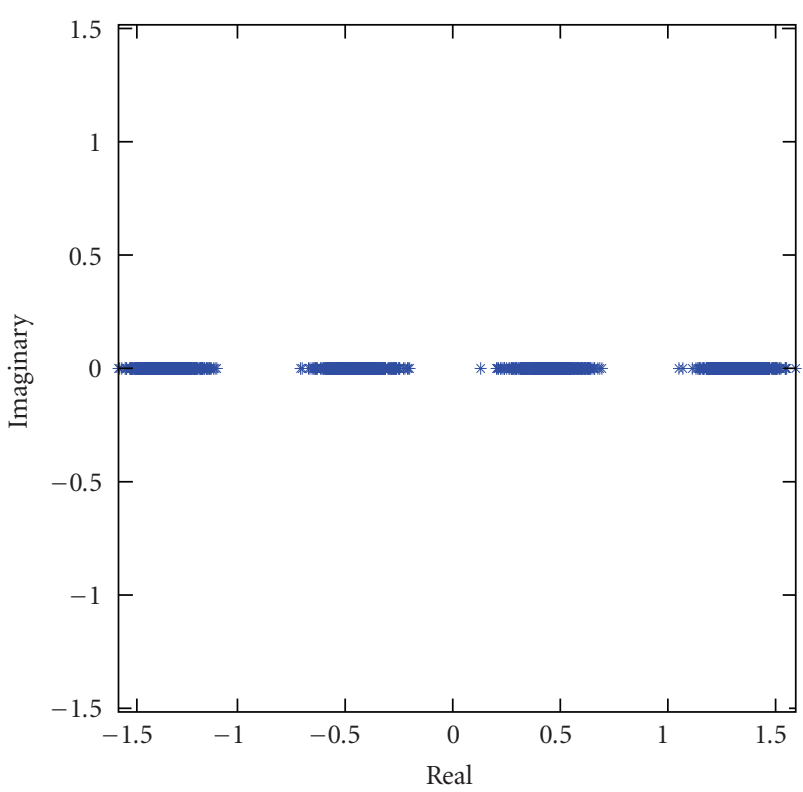

(a)

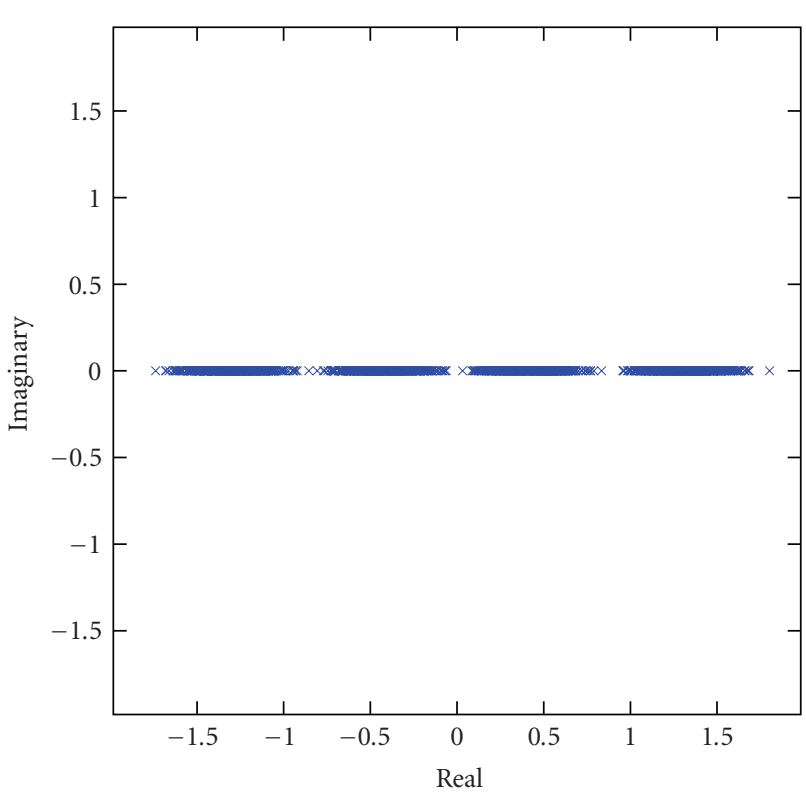

(b)

FIGURE 8: 4-PAM constellations after equalization using (a) GPS or (b) CMA2.

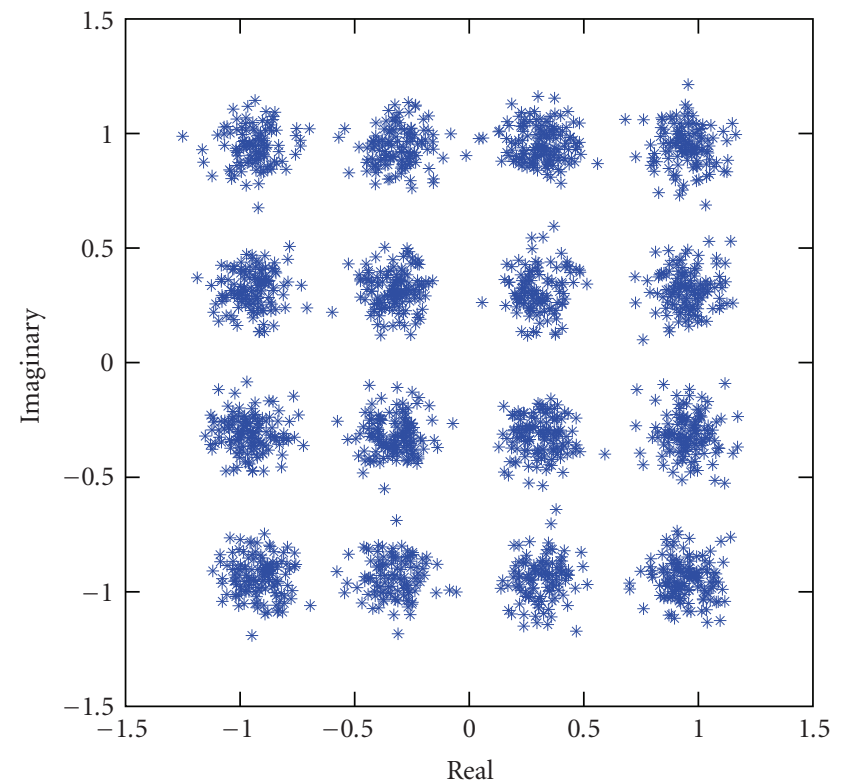

(a)

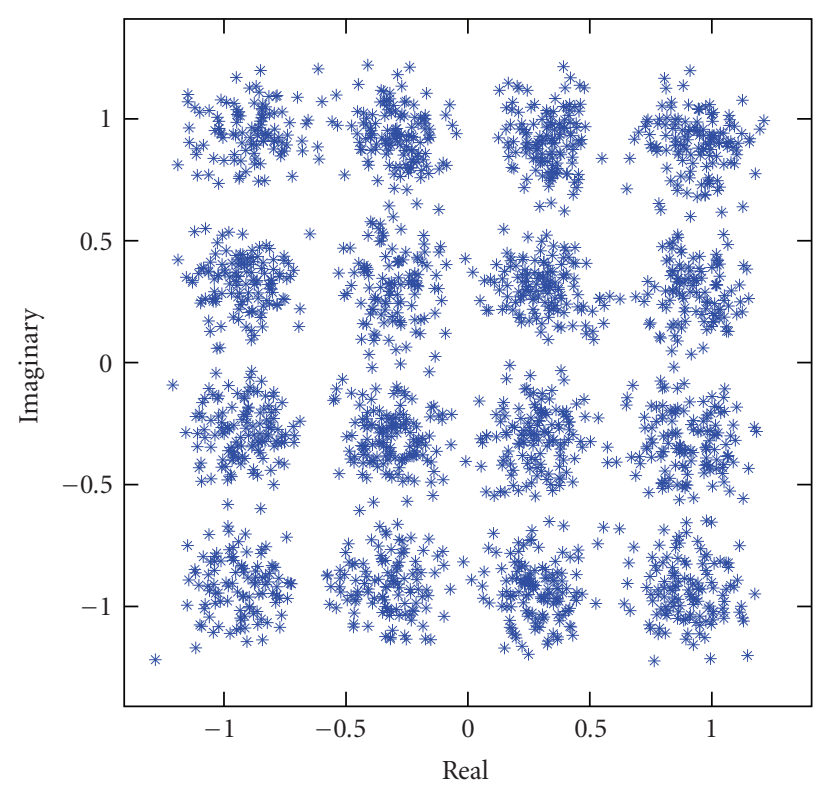

(b)

FIgURE 9: 16-QAM constellations after equalization using (a) GPS or (b) CMA2.

fact, the search step relies on the exploration of a large number of mesh points around the current point which is computational and time consuming. This phase is therefore omitted in the present work. On the contrary, the local poll step only explores the neighborhood of the current iteration on the mesh. This set of points $P_{k}$ is called the poll set and is defined by [24-26]

$$
P_{k}=\left\{\mathbf{w}_{k}+\Delta_{k} \mathbf{d}_{k}: \mathbf{d}_{k} \in \mathbf{D}_{k} \subseteq \mathbf{D}\right\},
$$

where $\Delta_{k}>0$ is the mesh size parameter that controls the fitness of the mesh, $\mathbf{w}_{k}$ the current $k$ th blind equalizer vector and $\mathbf{D}_{k}$ is a positive spanning set of directions $\mathbf{d}_{k}$ taken from D.

At iteration $k$, in order to find some point belonging to $P_{k}$ where the inequality $f\left(\mathbf{w}_{k}+\Delta_{k} \mathbf{d}_{k}\right)<f\left(\mathbf{w}_{k}\right)$ is verified, the poll phase is carried out by evaluating the fitness function that we need to optimize (namely, $f$ ) around the current blind equalizer vector $\mathbf{w}_{k}$. If such an improved mesh point 


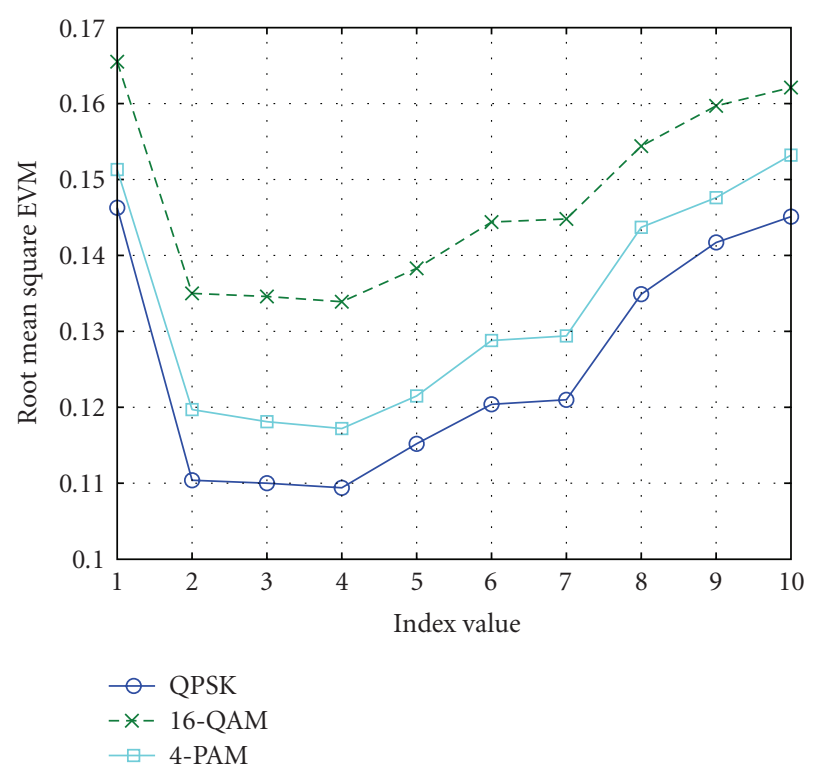

FIGURE 10: Averaged r.m.s. EVM values for CMA with various spike initializations.

(that decreases the fitness value) is found, then the iteration $k$ is called successful; otherwise it is considered unsuccessful. If the iteration is successful, the improved mesh point becomes the new iterate. This is achieved by setting $\mathbf{w}_{k+1}=\mathbf{w}_{k}+\Delta_{k} \mathbf{d}_{k}$. In this case, the mesh size parameter $\Delta_{k}$ is increased using the following updating rule:

$$
\Delta_{k+1}=\min \left(\tau \Delta_{k}, \Delta_{\max }\right),
$$

where $\tau>1$ is a step increase factor (often taken equal to 2), $\Delta_{0}$ is the initial step size, and $\Delta_{\max }$ is the maximum step size. The $\min (\cdot)$ function is used to ensure an upper limit to step size expansion.

On the other side, if no improved mesh point is found in all the poll step around $P_{k}$, the vector $\mathbf{w}_{k}$ is said to be a mesh local optimizer and is retained as the new iterate $\mathbf{w}_{k+1}=\mathbf{w}_{k}$. Moreover, the mesh size parameter decreases following the equation:

$$
\Delta_{k+1}=\max \left(\frac{\Delta_{k}}{\tau}, \Delta_{\min }\right),
$$

where the $\max (\cdot)$ function ensures that the exploration step does not get lower than a minimum step size $\Delta_{\min }$.

The process is repeated until a suitable stopping criterion is satisfied (maximum number of iterations exceeded or step size lower than the tolerance limit). The GPS algorithm is summarized in Algorithm 1 [27, 28].

\section{SIMULATION RESULTS}

The validity of the proposed method has been studied using simulation. We consider the, assumed unknown, real baud spaced channel:

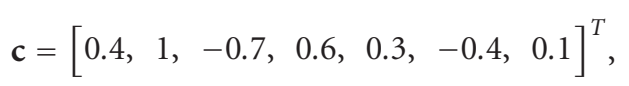

which is the same channel used in [6].

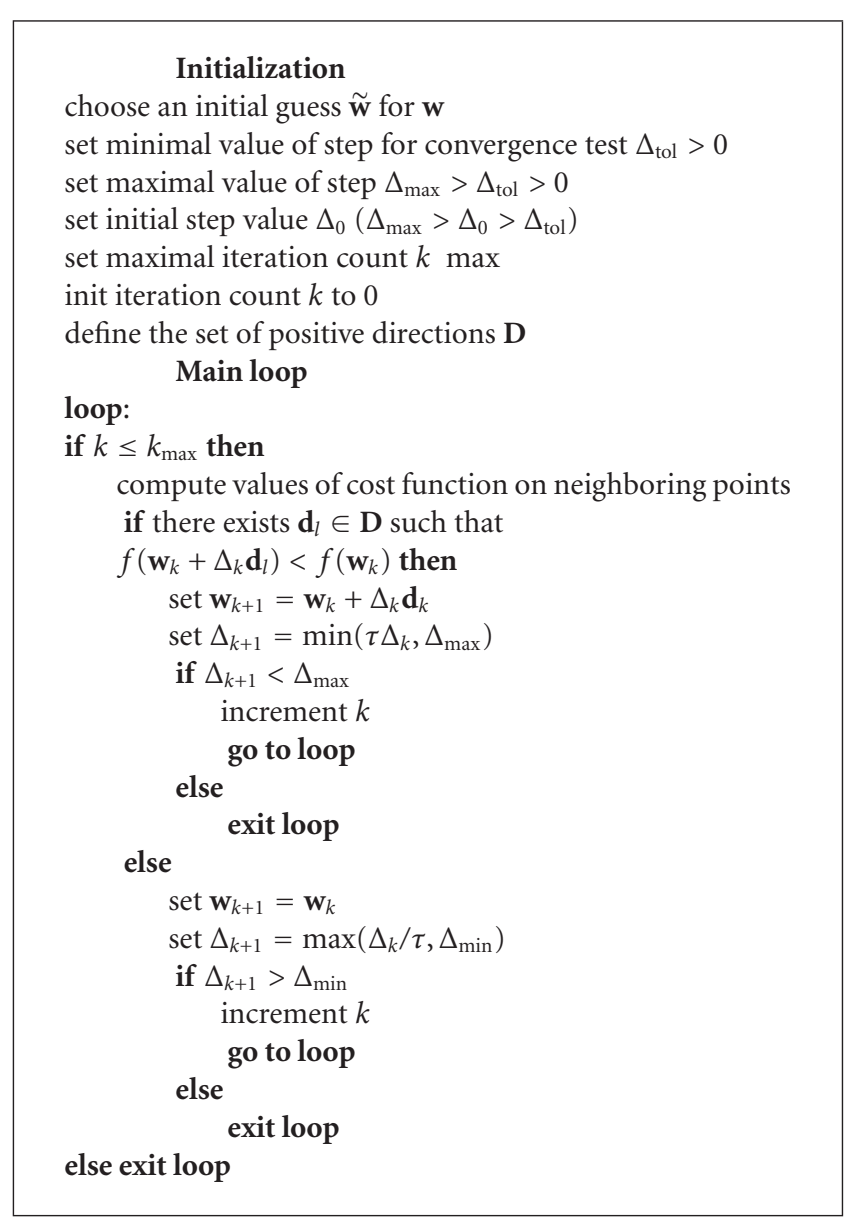

Algorithm 1: The algorithm for GPS optimization.

The corresponding magnitude and phase versus frequency characteristics, together with the $z$-plane zero pattern, are plotted in Figure 3. Note that the magnitude frequency response of this channel undergoes one severe fading (see Figure 3(a) top) and its corresponding phase is nonlinear (see Figure 3(a) bottom). Moreover this channel is mixed phase with four zeros inside the unit circle and one outside as highlighted in Figure 3(b).

We start by applying the GPS algorithm to the constrained blind equalization problem depicted in (7). The used input sequence is an i.i.d. unit power quadrature phase shift keying (QPSK) signal with a length of 2000 samples and the simulation parameters are as follows: the signal to noise ratio (SNR) is set to $20 \mathrm{~dB}$, the blind equalizer is a FIR baud spaced equalizer of length $L=20, \Delta_{0}=1$ (initial step size), $\Delta_{\min }=10^{-7}, \Delta_{\max }=10^{7}, \tau=2$. The value of $\tau$ taken here is the most often used in the literature and its only effect is to speed up or slow down convergence. The step related values play the role of stopping criteria: the min insures the precision of the converged value, the max alleviates fast divergence problems and the initial must take a reasonable value intermediate between both previous ones. Moreover, a maximum number of iterations has been fixed to 500, a value 
Table 1: Minimal cost using GPS for different delays around optimum.

\begin{tabular}{cccc}
\hline Delay index & Cost & Delay index & Cost \\
\hline 2 & -1.2513 & 3 & -1.2703 \\
4 & -1.2755 & 5 & -1.2743 \\
6 & -1.2717 & 7 & -1.2711 \\
\hline
\end{tabular}

which has been sufficient for most tries we performed on a number of different channels.

Since the selection of the fixed tap location is strictly related to the optimal delay selection problem and, assuming no a priori channel knowledge, we choose a linear constraint that fixes some tap to one at each iteration.

The probably suboptimal blind equalizer obtained after convergence is then used to estimate the desired optimal delay position using the CSR strategy as expressed in (12). Figure 4(a) shows the simulated estimates of the Wiener cost function for different delays from 0 to 25 . Let us note that this exceeds the equalizer filter length (taken equal to 20), but enables us to verify that a sufficiently high value has been chosen for it.

In Figure 4(a), the theoretical Wiener equalizer based on minimum square error (MSE) is given for the same delay positions. Let us remember that the MSE is defined as

$$
\operatorname{MSE} \triangleq E\left(\left|z_{k}-s_{k-\delta}\right|^{2}\right),
$$

and its corresponding optimal vector minimizer, namely, the Wiener equalizer is found as [13]

$$
\mathbf{w}^{\dagger}=\left(\mathbf{C}^{H} \mathbf{C}+\frac{\sigma_{n}^{2}}{\sigma_{s}^{2}} \mathbf{C}^{H} \mathbf{g}_{\delta^{\dagger}}\right)^{-1} \mathbf{C}^{H} \mathbf{g}_{\delta^{\dagger}},
$$

where $\mathbf{C}$ is the baud channel convolution matrix and $\delta^{\dagger}$ represents the desired optimal delay index, which corresponds to the index of the minimum diagonal element of $\mathbf{I}-\mathbf{C}\left(\mathbf{C}^{H} \mathbf{C}+\sigma_{n}^{2} \mathbf{I} / \sigma_{s}^{2}\right)^{-1} \mathbf{C}^{H}$.

It can be easily noticed from Figure 4(a) that both graphs have the same trend thus allowing the selection of the optimal delay which corresponds to an index $\delta^{\dagger}=4$. The value of the optimal delay is more clearly evidenced in Figure 4(b), which presents essentially the same data as Figure 4(a) for the MSE optimal equalizers, but in logarithmic scale. However, due to the occurrence of negative values for the simulated estimates of the Wiener cost function in Figure 4(a), full logarithmic representation is not truly feasible. Thus, the exact simulated values are given in Table 1 for index values around the optimum $\delta^{\dagger}$.

The negative values found for the Wiener cost function estimates result from the imposed blind equalizer linear constraint that fixes one tap to one. In fact, the zero forcing joint channel-equalizer impulse response has its important tap situated exactly at that position where the coefficient is constrained to be one. This results in $\mathbf{w}_{\delta} \widehat{\mathbf{c}}>1$ in (11). The negative values are not then to be considered as reflecting better performances in comparison to the theoretical Wiener MSE, but quite the contrary. It is actually more evident from Table 1 that the estimated lowest cost value of -1.2755 corresponds to a delay index $\delta^{\dagger}=4$. This latter is in accordance with the theoretical Wiener optimal delay $\delta^{\dagger}$. At present time, the constrained blind equalization problem can be reformulated more accurately as

$$
\text { minimize } f(\mathbf{w}) \text { subject to } w_{\delta^{+}}=w_{4}=1 .
$$

We apply GPS algorithm to this global optimization problem under the same simulation parameters (just stated above) but for different values of SNRs and different regressor sequence lengths $N$. The measured performance will be the intersymbol interference ISI, redefined below in logarithmic scale ( $\mathrm{dB}$ values) as

$$
\operatorname{ISI}(\mathrm{dB}) \triangleq 10 \log _{10}\left(\frac{\left[\sum_{i}\left|g_{i}\right|^{2}\right]-|g|_{\max }^{2}}{|g|_{\max }^{2}}\right) .
$$

Each simulation is run 30 times and the corresponding averaged results values are given in Figure 5. It can be noticed that the global blind equalization performs well for values of $N \geq 1000$ and is comparable to the Wiener equalizer for $N=2000$.

For performance comparison with the constant modulus algorithm, we use the BERGulator software for CMA simulations which may be downloaded from http://bard.ece.cornell.edu/downloads/. Figure 6 shows the CMA simulated performances in terms of ISI for different SNR values and three single spike initialization strategies, that we denote by CMA0, CMA1, and CMA4 the numerical values 0,1 , and 4 standing for the index of the unique nonzero blind equalizer tap in the initialization vector. The simulation parameters, the modulation type, the unknown channel, and the blind equalizer length, are the same as before; the fixed step size is $\mu=5 \times 10^{-4}$ and the iteration number is set to $2 \times 10^{4}$ to ensure final convergence.

It can be easily seen that, unlike the proposed algorithm which ensures global convergence behavior for sufficient samples sequence length, CMA is extremely vulnerable to the way of selecting the initial blind equalizer vector and local convergence is more likely to happen. This latter point is clearly highlighted in the case of CMA0 and CMA1. Moreover, the optimal Wiener delay index (which is in our case 4) corresponds exactly to the optimal position of the non-null element of CMA4 initial vector and also to the position of the equalizer tap constrained to one in the proposed algorithm. It may be noticed that CSR may equally well be applied to CMA, with the result of selecting the CMA4 case after initialization.

Furthermore, the proposed global blind optimizationbased algorithm outperforms significantly CMA in terms of local convergence properties and gives slightly better global performance than CMA4 (that is also CMA with CSR), especially in low-noise environments ( $\mathrm{SNR} \geq 30 \mathrm{~dB}$ ).

Figure 7 represents the constellations obtained for QPSK modulation, with SNR $=20 \mathrm{~dB}$ at receiver input. Let us notice that these constellations have been normalized, the baseband received signal modulus being taken as unity. It is clearly seen that the constellations points are not 
TABLE 2: Averaged r.m.s. EVM values using the proposed algorithm and CMA with optimum delay index $\delta^{\dagger}$, for three modulation types.

\begin{tabular}{lcc}
\hline Modulation type & GPS & Optimum CMA \\
\hline QPSK & 0.1090 & 0.1094 \\
16-QAM & 0.1243 & 0.1339 \\
4-PAM & 0.0997 & 0.1181 \\
\hline
\end{tabular}

resolved before equalization and become distinguishable for $\mathrm{CMA} 0$ and more separated for CMA1. A constellation phase rotation effect may also be noticed for CMA0 and, to a lesser extent, CMA1. Very satisfactory results are obtained with our proposed algorithm using GPS, these for CMA4 being visually quite identical. In fact, one approaches the Wiener optimum solution in both cases.

Other modulation types have been investigated. Figures 8 and 9 show constellations (normalized such that the baseband signal power is equal to unity) obtained for, respectively, 4-level pulse amplitude modulation (4-PAM) and 16-level quadrature amplitude modulation (16-QAM).

Only results from our GPS-based algorithm (a little better than those obtained with CMA4) and with CMA2 are shown for comparison purposes (constellations before equalization and using a CMA1 equalizer are not shown here).

The good performance of our algorithm is again evidenced. It may be noticed that, as may be logically expected, the same value is obtained for $\delta^{\dagger}$ independently of the modulation type.

Apart from constellation rotation, a measure of the equalizer efficiency is obtained using error vector magnitude (EVM) $[29,30]$. The root mean square (r.m.s.) EVM is defined as

$$
\mathrm{EVM}=\sqrt{\frac{\sum_{i=1}^{N}\left(\Delta I_{i}^{2}+\Delta Q_{i}^{2}\right)}{N \sum_{i=1}^{N}\left(I_{0, i}^{2}+Q_{0, i}^{2}\right)},}
$$

where $N$ is the number of emitted symbols, $I_{0, i}$ and $Q_{0, i}$, are the inphase and quadrature components, respectively, of the reference (noiseless) signal, $\Delta I_{i}=I_{i}-I_{0, i}, \Delta Q_{i}=Q_{i}-Q_{0, i}, I_{i}$ and $Q_{i}$ being the inphase and quadrature components of the received (noisy) signals.

Our algorithm has been run 30 times on sequences of 2000 emitted QPSK, 16-QAM, or 4-PAM symbols and 2000 added noise samples with $20 \mathrm{~dB}$ SNR, to get the averaged r.m.s. EVM values given in Table 2 (the averaging process is taken over a sufficiently high number of samples as per Monte Carlo method).

The simulation has been repeated using CMA and variable spike initializations for comparison purposes. The EVM results are shown in Figure 10 versus delay index value around optimum (from 1 to 10 ) for the three previously used modulation types.

The corresponding minimum cost function values (for delay index $\delta^{\dagger}$ ) are also given in Table 2 .

Not surprisingly, one sees that the performance decreases for higher efficiency 16-QAM modulation. Moreover 4-PAM and 16-QAM are not constant envelope modulations and thus CMA is not well-suited for them. As a consequence, our GPS-based algorithm outperforms noticeably CMA in these two cases. It has also been noticed during the simulation that our algorithm gives much lesser dispersion in EVM values when compared to CMA (lower variance).

\section{DISCUSSION AND CONCLUSION}

In this paper, a baud spaced blind equalization method based on GPS and CSR has been presented in detail and compared to the CMA algorithm. Successful simulation results have been obtained on a number of different, real, or complex channels. For example, real static channel presenting a single deep fading and mixed phase has been presented. We have shown the good performances of the proposed equalizer, even for nonconstant envelope modulations. For constant envelope modulations, the performances are nearly identical to that given by CMA, after selecting the optimum CMA spike delay value for its initialization vector and correctly choosing its step size. This has also been verified for QPSK as reported here and noted for 8-PSK and 16-PSK. Other static channels with more than one fading have also been tested, with essentially the same conclusions as above.

Our algorithm involves unavoidable steps of cost function computation (as any other equalization one) and simple algebraic equations for updating the equalizer weights (no gradient computation), testing, and loop instructions. It may be implemented in a FPGA-floating point DSP structure, owing to its reasonable complexity. For performance evaluation, the main concern is the number of required cost function evaluations (which depends on the speed of convergence, and thus equalized channel and initial conditions). The comparison with CMA algorithm using CSR initialization, for a number of different channels, leads to the conclusion that the number of cost function evaluations is of the same order of magnitude as the CSRCMA and our algorithm, with a little to significant advantage for the latter in the cases of channels with problems (like amplitude or frequency selectivity) or of nonconstant modulus modulations.

Our future work will be directed to extending our algorithm to fractionally spaced equalization, improving the CSR step, and using space diversity. Moreover, the case of slowly varying channels will be considered.

\section{ACKNOWLEDGMENT}

The authors thank Dr. Walaa Hamouda from the Department of Electrical and Computer Engineering of Concordia University for helpful comments and suggestions that have led to an improved paper.

\section{REFERENCES}

[1] J. Zhu, X.-R. Cao, and R.-W. Liu, "A blind fractionally spaced equalizer using higher order statistics," IEEE Transactions on Circuits and Systems II, vol. 46, no. 6, pp. 755-764, 1999. 
[2] F. Alberge, P. Duhamel, and M. Nikolova, "Adaptive solution for blind identification/equalization using deterministic maximum likelihood," IEEE Transactions on Signal Processing, vol. 50, no. 4, pp. 923-936, 2002.

[3] J.-P. Delmas, H. Gazzah, A. P. Liavas, and P. A. Regalia, "Statistical analysis of some second-order methods for blind channel identification/equalization with respect to channel undermodeling," IEEE Transactions on Signal Processing, vol. 48, no. 7, pp. 1984-1998, 2000.

[4] C.-Y. Chi, C.-Y. Chen, C.-H. Chen, and C.-C. Feng, "Batch processing algorithms for blind equalization using higherorder statistics," IEEE Signal Processing Magazine, vol. 20, no. 1, pp. 25-49, 2003.

[5] C.-H. Chen, C.-Y. Chi, and W.-T. Chen, "New cumulant-based inverse filler criteria for deconvolution of nonminimum phase systems," IEEE Transactions on Signal Processing, vol. 44, no. 5, pp. 1292-1297, 1996.

[6] M. Kawamoto, M. Ohata, K. Kohno, Y. Inouye, and A. K. Nandi, "Robust super-exponential methods for blind equalization in the presence of Gaussian noise," IEEE Transactions on Circuits and Systems II: Express Briefs, vol. 52, no. 10, pp. 651-655, 2005.

[7] A.G. Bessios and C. L. Nikias, "POTEA: the power cepstrum and tricoherence equalization algorithm," IEEE Transactions on Communications, vol. 43, no. 11, pp. 2667-2671, 1995.

[8] H. Mathis and S. C. Douglas, "Bussgang blind deconvolution for impulsive signals," IEEE Transactions on Signal Processing, vol. 51, no. 7, pp. 1905-1915, 2003.

[9] B. Maricic, Z.-Q. Luo, and T. N. Davidson, "Blind constant modulus equalization via convex optimization," IEEE Transactions on Signal Processing, vol. 51, no. 3, pp. 805-818, 2003.

[10] R. Pandey, "Complex-valued neural networks for blind equalization of time varying channels," International Journal of Signal Processing, vol. 1, no. 1, pp. 1-8, 2005.

[11] V. Shtrom and H. Fan, "A refined class of cost functions in blind equalization," in Proceedings of the IEEE International Conference on Acoustics, Speech, and Signal Processing (ICASSP '97), vol. 3, pp. 2273-2276, Munich, Germany, April 1997.

[12] V. Shtrom and H. Fan, "New class of zero-forcing cost functions in blind equalization," IEEE Transactions on Signal Processing, vol. 46, no. 10, pp. 2674-2683, 1998.

[13] C. R. Johnson Jr., P. Schniter, T. J. Endres, J. D. Behm, D. R. Brown, and R. A. Casas, "Blind equalization using the constant modulus criterion: a review," Proceedings of the IEEE, vol. 86, no. 10, pp. 1927-1950, 1998.

[14] S. Chen, T. B. Cook, and L. C. Anderson, "A comparative study of two blind FIR equalizers," Digital Signal Processing, vol. 14, no. 1, pp. 18-36, 2004.

[15] S. Vembu, S. Verdu, R. A. Kennedy, and W. Sethares, "Convex cost functions in blind equalization," IEEE Transactions on Signal Processing, vol. 42, no. 8, pp. 1952-1960, 1994.

[16] A. T. Erdogan and C. Kizilkale, "Fast and low complexity blind equalization via subgradient projections," IEEE Transactions on Signal Processing, vol. 53, no. 7, pp. 2513-2524, 2005.

[17] L. Tong and H. H. Zeng, "Channel surfing reinitialization for the constant modulus algorithm," IEEE Signal Processing Letters, vol. 4, no. 3, pp. 85-87, 1997.

[18] S. Evans and L. Tong, "Online adaptive reinitialization of the constant modulus algorithm," IEEE Transactions on Signal Processing, vol. 48, no. 4, pp. 537-539, 2000.

[19] H. H. Zeng, L. Tong, and C. R. Johnson Jr., "Relationships between the constant modulus and wiener receivers," IEEE Transactions on Information Theory, vol. 44, no. 4, pp. 15231538, 1998.
[20] V. Torczon, "On the convergence of pattern search algorithms," SIAM Journal on Optimization, vol. 7, no. 1, pp. 1-25, 1997.

[21] C. Davids, "Theory of positive linear dependence," American Journal of Mathematics, vol. 76, no. 4, pp. 733-746, 1954.

[22] E. Polak and M. Wetter, "Generalized pattern search algorithms with adaptive precision function evaluations," Tech. Rep. LBNL-52629, Lawrence Berkeley National Laboratory, Berkeley Calif, USA, May 2003.

[23] E. D. Dolan, R. M. Lewis, and V. Torczon, "On the local convergence of pattern search," SIAM Journal on Optimization, vol. 14, no. 2, pp. 567-583, 2003.

[24] M. A. Abramson, "Second-order behavior of pattern search," SIAM Journal on Optimization, vol. 16, no. 2, pp. 515-530, 2006.

[25] C. Audet and J. E. Dennis Jr., "A pattern search filter method for nonlinear programming without derivatives," SIAM Journal on Optimization, vol. 14, no. 4, pp. 980-1010, 2004.

[26] M. A. Abramson, C. Audet, and J. E. Dennis Jr., "Generalized pattern searches with derivative information," Mathematical Programming, vol. 100, no. 1, pp. 3-25, 2004.

[27] A. Zaouche, I. Dayoub, and J. M. Rouvaen, "Blind constant modulus equalization using hybrid genetic algorithm and pattern search optimization," in Proceedings of the 7th World Wireless Congress (WWC '06), pp. 37-42, San Francisco, Calif, USA, May 2006.

[28] A. Zaouche, I. Dayoub, and J. M. Rouvaen, "Blind equalization via the use of generalized pattern search optimization and zero forcing sectionnally convex cost function," in Proceedings of the 2nd IEEE International Conference on Information and Communication Technologies (ICTTA '06), vol. 2, pp. 23032308, Damaskus, Syria, April 2006.

[29] K. M. Voelker, "Apply error vector measurements in communications design," Microwaves \& RF, pp. 143-152, December 1995.

[30] R. Hassun, M. Flaherty, R. Matreci, and M. Taylor, "Effective evaluation of link quality using error vector magnitude techniques," in Proceedings of the Annual Wireless Communications Conference, pp. 89-94, Boulder, Calif, USA, August 1997. 